\title{
Airflow resistivity of models of fibrous acoustic materials
}

\section{Tarnow, Viggo}

\section{Published in:}

Acoustical Society of America. Journal

Link to article, DOI:

10.1121/1.417233

Publication date:

1996

\section{Document Version}

Publisher's PDF, also known as Version of record

Link back to DTU Orbit

Citation (APA):

Tarnow, V. (1996). Airflow resistivity of models of fibrous acoustic materials. Acoustical Society of America. Journal, 100(6), 3706-3713. https://doi.org/10.1121/1.417233

\section{General rights}

Copyright and moral rights for the publications made accessible in the public portal are retained by the authors and/or other copyright owners and it is a condition of accessing publications that users recognise and abide by the legal requirements associated with these rights.

- Users may download and print one copy of any publication from the public portal for the purpose of private study or research.

- You may not further distribute the material or use it for any profit-making activity or commercial gain

- You may freely distribute the URL identifying the publication in the public portal

If you believe that this document breaches copyright please contact us providing details, and we will remove access to the work immediately and investigate your claim 


\title{
Airflow resistivity of models of fibrous acoustic materials
}

Viggo Tarnow

Department of Applied Engineering Design and Production, Technical University of Denmark, Bygning 358, DK 2800 Lyngby, Denmark

(Received 2 November 1995; revised 28 May 1996; accepted 11 July 1996)

\begin{abstract}
A new way of calculating the airflow resistivity of randomly placed parallel cylinders is presented. The calculation is based on Voronoi polygons, and the resistivity is given by the mean spacing between the fibers, their diameters, and the physical properties of air. New explicit formulas for the resistivity are given, which are valid for the cylinder (fiber) concentrations found in acoustic materials. A one-dimensional model consisting of parallel plates with random spacing between the plates is first discussed. Then a two-dimensional model consisting of parallel cylinders randomly spaced is treated for flow parallel and perpendicular to the cylinders. The resistivity formulas are exact for plates and approximate for cylinders. (C) 1996 Acoustical Society of America.
\end{abstract}

PACS numbers: 43.58.Bh, 43.20.Hq, 43.20.Jr, 43.55.Ev [SLE]

\section{LIST OF SYMBOLS}

\section{$A, A_{0}, A_{4} \quad$ integration constants}

a radius of fiber or cylinder

$b \quad$ mean spacing between plates or square root of area per fiber

d volume concentration of cylinders

$K \quad$ permeability

$l \quad$ length of a sample of fiber material or length of a cylinder

n normal to a closed surface that points away from the enclosed volume

power to a cell

pressure

probability

resistivity of fiber material

resistivity of fiber material for longitudinal flow through a square lattice

$R_{\mathrm{lr}} \quad$ resistivity of fiber material for longitudinal flow through a random lattice

$R_{\mathrm{cs}} \quad$ resistivity of fiber material for cross flow through a square lattice

$R_{\text {cr }} \quad$ resistivity of fiber material for cross flow through a random lattice $r$

$S$

$u$

$v$

$x$

$y$

$z$

$\Delta p$

$\eta$

$\xi$

$\rho$

$\sigma$

$\phi$

$\Psi$

$\Psi_{1}$

$\psi$

$\omega$

$\langle u\rangle$

$\langle\nabla p\rangle$ vector from origin of coordinate system to point in air

area of Voronoi cell

air velocity

angle

coordinate

coordinate

coordinate

pressure decrease

viscosity of air

spacing between plates

radius of cylinder equivalent to cell

cross-section area of cell divided by average area of cell

porosity

volume flux of air (volume current)

volume flux of air per unit length

Stokes' stream function

vorticity

mean velocity

mean pressure gradient

\section{INTRODUCTION}

Sound velocity and attenuation in fiber materials such as glass wool are mainly determined by the constant velocity air flow resistivity, as is well known from the work of Delany and Bazley. ${ }^{1}$ If one knows the flow resistivity one can predict the acoustical properties of glass wool. It is of academical and practical interest to be able to calculate the flow resistivity of fiber materials from the fiber diameters and their density and distribution in space. As an introduction to the more difficult case of parallel cylinders with random spacing, the flow resistivity for parallel plates with random spacings is calculated. Then parallel cylinders are treated both for airflow parallel and perpendicular to the cylinders. Similar results have been used to explain measurements on glass wool $\left(\right.$ see Tarnow $\left.^{2}\right)$.

The flow resistivity is defined by an experiment where a

sample of the fiber material is placed in a tube. A steady airflow is sent through the sample. The mean air velocity $\langle u\rangle$, the pressure decrease over the sample $\Delta p$, and the length of the sample $l$ are measured. The resistivity of the material is defined as the pressure drop per length divided by the mean air velocity:

$$
R=\Delta p /\langle u\rangle l .
$$

In all the models the procedure of calculation is the same: The linearized Navier-Stokes equation for the velocity is solved either exactly or approximately with the boundary conditions given by the geometry of the problem. From the velocity and the pressure gradient the resistivity is found. In a later paper the dynamic flow resistivity will be calculated. 
Flow resistivity of cylinders in regular arrays has been treated in the literature by many authors, as can be seen in the review by Aström et al. ${ }^{3}$ However, there are only few papers on the more difficult problem of randomly placed cylinders, such as Howells ${ }^{4}$ and Sangani and Yao, ${ }^{5}$ and these authors do not provide explicit formulae for the resistivity; unlike the present paper.

In order to compute the acoustical properties of fiber materials one also needs the compressibility of air between fibers. This has been presented in Tarnow. ${ }^{6}$

\section{GENERAL THEORY}

The Navier-Stokes equation for incompressible stationary flow can be found in Landau and Lifshitz. ${ }^{7}$ We assume the air velocity is small, i.e., low Reynolds number. In this case the equation for the velocity $\mathbf{u}$ is

$$
\eta \nabla^{2} \mathbf{u}=\boldsymbol{\nabla} p,
$$

where $p$ the pressure and $\eta$ the viscosity of air, which is $\eta=1.846 \times 10^{-5} \mathrm{~kg} /(\mathrm{m} \mathrm{s})$ at the temperature $27^{\circ} \mathrm{C}$. In Pierce ${ }^{8}$ the temperature dependence of the viscosity is given. The incompressibility of the flow is expressed by

$$
\boldsymbol{\nabla} \cdot \mathbf{u}=0 .
$$

These equations are to be solved with the boundary condition that the velocity at the cylinders equals zero.

\section{ONE-DIMENSIONAL MODELS}

A one-dimensional model having a resemblance to real fiber materials consists of sets of parallel plates of solid material. Between the plates air flows parallel with the plates driven by a constant pressure gradient.

We use a rectangular coordinate system. The $x$ axis is perpendicular to the plates. The $z$ axis is in the direction of the flow $\left(\partial p / \partial x=\partial p / \partial y=0\right.$, and $\left.u_{x}=u_{y}=0\right)$. A differential equation for the velocity follows from Eq. (2). In this case Eq. (3) is fulfilled automatically, and one gets

$$
\frac{\partial^{2} u_{z}}{\partial x^{2}}=\frac{1}{\eta} \frac{\partial p}{\partial z},
$$

where $\partial p / \partial z=-\Delta p / l$.

The resistivity depends on the spacings between the plates. Two models are considered, the first one with equal spacings between plates, and the second one with random spacings.

\section{A. Regular lattice with constant plate thickness}

We consider a set of parallel plates with equal spacings, $b$, between neighbor plates (Fig. 1, upper part). This case has been treated by many authors, e.g., Landau and Lifshitz, ${ }^{7}$ but it is considered here as an introduction to the new case of random placement of plates. The plates are assumed to be infinitely thin. The velocity that satisfies Eq. (4) and is zero on the plates is

$$
u_{z}=\frac{1}{2 \eta} \frac{\partial p}{\partial z}\left(x^{2}-b x\right)
$$
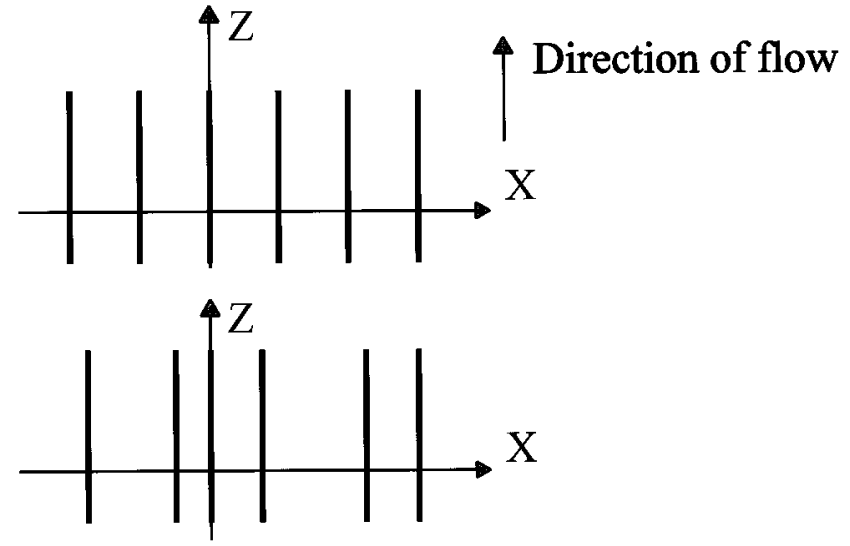

FIG. 1. Parallel plates with flow in the direction of the $z$ axis. The upper part of the figure is for regular spacing, the lower one for random spacing.

where $x$ is measured from one plate. The flux of air (volume current) between the two plates is found by integration of the velocity over the spacings between the plates. The velocity does not depend on the $y$ coordinate, so the integration in this direction will be omitted. The flux per unit distance in the $y$-direction $\Psi_{1}$, can be found by $\Psi_{1}=\int_{0}^{b} u_{z} d x$. The result is

$$
\Psi_{1}=-\frac{1}{12 \eta} \frac{\partial p}{\partial z} b^{3} .
$$

The mean velocity $\left\langle u_{z}\right\rangle$ equals this flux divided by the spacing between the plates, thus

$$
\left\langle u_{z}\right\rangle=\Psi_{1} / b .
$$

The resistivity as defined in Eq. (1) is found from (6) and (7),

$$
R=12 \eta / b^{2} .
$$

\section{B. Random spacings between plates}

A set of parallel plates with random spacings is considered in Fig. 1, lower part. This is of interest as an introduction to the theory of the resistivity of real fiber materials. We consider the simplest case where the plates are infinitely thin.

The plates are placed randomly with a mean spacing of $b$. We assume for the sake of simplicity that the spacings between two neighbor plates have Poisson distribution. The probability $d q$ of finding the spacing in the interval from $\xi$ to $\xi+d \xi$ is according to Ziman, ${ }^{9}$

$$
d q=\frac{1}{b} e^{-\xi / b} d \xi
$$

First we calculate the ensemble average flux $\left\langle\Psi_{1}\right\rangle$ between two plates from Eq. (6):

$$
\left\langle\Psi_{1}\right\rangle=-\frac{1}{12 \eta} \frac{\partial p}{\partial z} \int_{0}^{\infty} \xi^{3} e^{-\xi / b} d \xi=-\frac{2 b^{3}}{\eta} \frac{\partial p}{\partial z} .
$$

This is divided by $b$ to give the mean velocity, and from the definition Eq. (1), the resistivity is found:

$$
R=2 \eta / b^{2} .
$$


It is very interesting to compare this with the resistivity of regular spaced plates with zero thickness [Eq. (8)]. The resistivity of the regular spaced plates is much higher than the resistivity of the random spaced plates with the same density. This is easy to understand. The flux between two plates depends on their spacings in the third power. Therefore, the flow is much higher between distant plates than between close ones with the same pressure gradient. If one regards a set of many randomly distributed plates, one sees that there is a certain number of plates with a large spacing between them. Between these plates the air flows easily. The flow in a set of many plates is much higher if the plates are randomly distributed. The set of regularly spaced plates do not have these open parts. Therefore, the resistivity of equally spaced plates is much higher than the resistivity of randomly spaced plates.

This can be illustrated if we think of a set of plates where all of the plates are placed together with zero spacing between them. In this case the air flows easily and the resistivity is very small.

\section{FLOW PARALLEL WITH FIBERS}

A model of fibrous materials consisting of parallel cylinders with equal radius and flow perpendicular to the cylinders will be addressed. First, the case of cylinders arranged in a square lattice will be discussed. Then the case of random distribution of fibers will be discussed. The volume concentration of fibers is quite small for glass fiber materials, in most cases less than 0.02 . Therefore, we only need theoretical results for low density of cylinders.

In Landau and Lifshitz ${ }^{7}$ it is shown that the pressure gradient is a constant for flow parallel to cylinders $(\partial p / \partial x$ $\left.=\partial p / \partial y=0, u_{x}=u_{y}=0\right)$, and that the velocity may be computed from the equation

$$
\eta \nabla^{2} u_{z}=\frac{\partial p}{\partial z}
$$

where we use a coordinate system with the $z$ axis in the direction of the cylinders.

\section{A. Square lattice}

Sparrow and Loeffler ${ }^{10}$ have treated the case of flow parallel with parallel cylinders placed in a regular square lattice. They use polar coordinates and write the velocity to be computed from Eq. (12) as series,

$$
\begin{aligned}
u_{z}= & \frac{1}{4 \eta} \frac{\partial p}{\partial z}\left(r^{2}-a^{2}\right)+A_{0} \ln \left(\frac{r}{a}\right)+A_{4}\left(r^{4}-\frac{a^{8}}{r^{4}}\right) \cos (4 \nu) \\
& +\cdots,
\end{aligned}
$$

where $r$ is the radius vector, $\nu$ is the polar angle, $a$ is the radius of the cylinders, and $A_{0}$ and $A_{4}$ are constants to be determined by the boundary conditions. Due to the symmetry of the lattice we get

$$
\frac{\partial u_{z}}{\partial n}=0
$$

on the border of the square unit cell in Fig. 2, where $n$ is a normal to the border of the square. Sparrow and Loeffler ${ }^{10}$

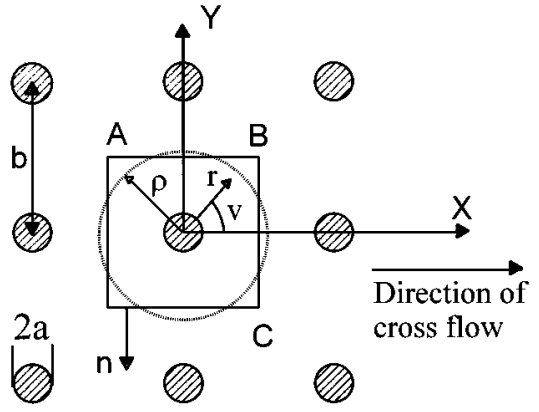

FIG. 2. Cylinders placed in a regular lattice. The square is the unit cell. The flow is perpendicular either to the paper or in the direction of the $x$ axis. The broken line is a circle with the same area as the unit cell. The circle with proper boundary conditions is a replacement for the square on which it is more difficult to fulfill the boundary conditions.

chose the constants so that this boundary condition was fulfilled. For volume concentration less than 0.1 their results show that the resistivity can be computed with good accuracy by the following procedure.

The velocity can be found by replacing the square on Fig. 1 by a circle with the same area as the square, and require that on the circle

$$
\frac{\partial u_{z}}{\partial r}=0
$$

The velocity that fulfils Eq. (12) and the boundary conditions is

$$
u_{z}=\frac{1}{\eta} \frac{\partial p}{\partial z}\left[\frac{1}{4}\left(r^{2}-a^{2}\right)-\frac{\rho^{2}}{2} \ln \frac{r}{a}\right],
$$

where $\rho$ is the radius of the circle. The volume flux $\Psi$ between the two concentric circles is $\Psi=\int_{a}^{\rho} u_{z} 2 \pi r d r$. By substituting Eq. (16) into this and performing the integration, one gets

$$
\begin{aligned}
\Psi= & \frac{1}{\eta} \frac{\partial p}{\partial z}\left[\frac{3}{8} \pi \rho^{4}-\frac{1}{2} a^{2} \pi \rho^{2}-\frac{1}{2} \pi k \rho^{4} \ln \left(\frac{\rho}{a}\right)\right. \\
& \left.+\frac{1}{8} \pi a^{4}\right] .
\end{aligned}
$$

We introduce the area $S=\pi \rho^{2}$ of the circle, and write the flux in terms of $S$,

$$
\begin{aligned}
\Psi= & \frac{1}{\eta} \frac{\partial p}{\partial z}\left[\frac{3}{8 \pi} S^{2}-\frac{1}{2} a^{2} S-\frac{S^{2}}{4 \pi} \ln \left(\frac{S}{\pi a^{2}}\right)\right. \\
& \left.+\frac{1}{8 \pi}\left(\pi a^{2}\right)^{2}\right] .
\end{aligned}
$$

The area of the circle is set equal to the area of the unit cell of the square lattice. If we call the length of the side of the unit cell $b$, we get

$$
S=b^{2} \text {. }
$$

The mean velocity is

$$
\left\langle u_{z}\right\rangle=\Psi / b^{2} .
$$

From the last two equations one gets the mean velocity 


$$
\begin{aligned}
\left\langle u_{z}\right\rangle= & -\frac{1}{4 \pi \eta b^{2}} \frac{\partial p}{\partial z}\left[\ln \left(\frac{b^{2}}{\pi a^{2}}\right)-\frac{3}{2}+\frac{2 \pi a^{2}}{b^{2}}\right. \\
& \left.-\frac{1}{2}\left(\frac{\pi a^{2}}{b^{2}}\right)\right] .
\end{aligned}
$$

This can be written in terms of $d$, the volume concentration of fibers

$$
d=\pi a^{2} / b^{2} .
$$

In acoustics the porosity $\phi$ is used. $\phi=1-d$ :

$$
\left\langle u_{z}\right\rangle=-\frac{1}{4 \pi \eta b^{2}} \frac{\partial p}{\partial z}\left[\ln \left(\frac{1}{d}\right)-\frac{3}{2}+2 d-\frac{1}{2} d^{2}\right] .
$$

From Eq. (1) one gets the resistivity,

$$
R_{\mathrm{ls}}=\frac{4 \pi \eta}{b^{2}\left[\ln (1 / d)-\frac{3}{2}+2 d\right]},
$$

where a term with $d^{2}$ has been neglected.

Sparrow and Loeffler's paper ${ }^{10}$ shows this procedure gives the right result for the resistivity in the lowconcentration limit. It is similar to the approximation of Wigner and Seitz used in solid-state theory for computing metallic cohesion (see Dekker ${ }^{11}$ ).

Berdichevsky and $\mathrm{Cai}^{12}$ have also treated parallel flow through parallel cylinders both analytically and numerically; their results, together with the results of Sparrow and Loeffler, ${ }^{10}$ indicate that for volume concentration less than 0.1 the resistivity of Eq. (24) has an accuracy better than $2 \%$. This can be seen from the following:

Berdichevsky and $\mathrm{Cai}^{12}$ use a permeability instead of the resistivity. They define a dimensionless permeability as

$$
K=\frac{\eta\langle u\rangle}{a^{2}\langle\nabla p\rangle},
$$

where $\langle u\rangle$ is the mean velocity and $\langle\nabla p\rangle$ is the mean gradient. The resistivity may be written in terms of the permeability

$$
R=\eta / a^{2} K
$$

If one takes their Eq. (4), neglects a term $d^{2}$, and set the result into Eq. (26), one gets exactly Eq. (24). Their Table I gives for a concentration of 0.1 the permeability 2.494 computed by their Eq. (4). By finite element calculations they find the permeability 2.552 .

\section{B. Random lattice}

We want to compute the resistivity of a lattice of parallel cylinders placed randomly for flow parallel with the fibers. We find the velocity by a method analogous to the one above by using the so-called Voronoi polygons.

Points placed randomly in a plane are considered, and we divide the plane into polygons in the following way: Draw lines from each point to the nearest-neighbor points, and draw normals that bisects these lines. The normals enclose an area around each point, as shown in Fig. 3. Aurenhammer ${ }^{13}$ has written a survey of Voronoi diagrams.

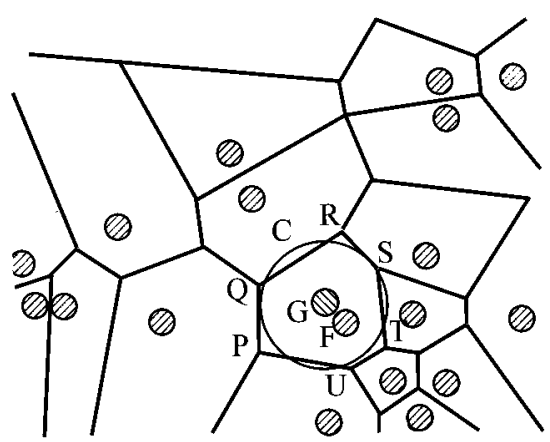

FIG. 3. The circles represent the parallel cylinders. The circles are placed randomly in the plane and are surrounded by Voronoi polygons. The flow is either perpendicular to the paper or in the plane of the paper. The circle $\mathrm{C}$ is a replacement for the polygon PQRSTU. The flow problem is solved for flow between the concentric circles $\mathrm{C}$ and $\mathrm{G}$, where $\mathrm{G}$ is the circle $\mathrm{F}$ moved to the center of $\mathrm{C}$. The vorticity is assumed to be zero on the circle $\mathrm{C}$, and the velocity is zero on $\mathrm{G}$.

We use the Voronoi polygons to compute the air velocity when the pressure gradient is given. The velocity gradient normal to the perimeter of a polygon around a lattice point must be close to zero. This quantity is exactly zero for a square lattice due to symmetry, and symmetry considerations show that the gradient of the velocity perpendicular to the polygons must be zero close to the polygons.

We replace the polygons with circles with the same area and assume that the lattice point is in the center of the polygon. The boundary conditions are that the velocity is zero at the inner circle and the gradient of the velocity is zero at the outer circle. This procedure gives an upper limit to the resistivity because the lattice point is assumed to be placed in the center of the circle. In some Voronoi cells this is not the case. The flow through a cell with a cylinder not in the center of a polygon must be higher than the flow through the same cell if the cylinder is in the center of the cell. Therefore, the computed resistivity is higher than the exact value. But the difference is small at low concentration because the resistance is determined by the shear stress at the inner cylinder, and this stress does not depend strongly on the eccentricity when the concentration is low [Because of Eq. (14), the shear stress is zero at the outer cylinder.] This is confirmed by comparison with numeric calculations quoted in the end this section.

The method of computation is the same as was used in the one-dimensional case. The average volume flux is computed for the Voronoi cells. This gives from Eq. (18)

$$
\begin{aligned}
\langle\Psi\rangle= & \frac{1}{\eta} \frac{\partial p}{\partial z}\left[\frac{3}{8 \pi}\left\langle S^{2}\right\rangle-\frac{1}{2} a^{2}\langle S\rangle-\frac{1}{4 \pi}\left\langle S^{2} \ln \left(\frac{S}{\pi a^{2}}\right)\right\rangle\right. \\
& \left.+\frac{1}{8 \pi}\left(\pi a^{2}\right)^{2}\right],
\end{aligned}
$$

where the angular brackets mean average, and $S$ is the area of the Voronoi cells.

The average of area of the Voronoi cells is

$$
\langle S\rangle=b^{2} \text {. }
$$

The average of the square of the area has been computed exactly. It is given in Weaire et al. ${ }^{14}$

$$
\left\langle S^{2}\right\rangle=1.280\langle S\rangle^{2} \text {. }
$$

Viggo Tarnow: Airflow resistivity/fibrous acoustic materials 3709 
We introduce the area of the Voronoi cells divided by the average area $\sigma$,

$$
\sigma=S / b^{2} .
$$

From (29) and (30) one gets

$$
\left\langle\sigma^{2}\right\rangle=1.280 \text {. }
$$

In order to compute the mean flux Eq. (27), we need

$$
\left\langle S^{2} \ln \left(\frac{S}{\pi a^{2}}\right)\right\rangle=\langle S\rangle^{2}\left\langle\sigma^{2} \ln (\sigma)\right\rangle-\langle S\rangle^{2} \ln (d),
$$

which was found by inserting $S$ from Eq. (30) into the lefthand side of (32) and using (22) and (28). The distribution function for $S$ is given in Andrade and Fortes. ${ }^{15}$ The probability $d q$ that the area of a Voronoi polygon is in the interval $S, S+d S$ is found from

$$
\frac{d q}{d S}=\frac{1}{\langle S\rangle} \frac{\alpha^{\alpha}}{\Gamma(\alpha)}\left(\frac{S}{\langle S\rangle}\right)^{\alpha-1} \exp \left(-\frac{\alpha S}{\langle S\rangle}\right),
$$

where $\alpha \approx 3.61$ and $\Gamma(\alpha)$ is the gamma function. It is a gamma distribution, which is used instead of the Poisson distribution for the parallel plates. This distribution of Andrade and Fortes ${ }^{15}$ does not take into account the fact that the cylinder cannot have axes closer than $2 a$. However, this is not important because the error is small when the volume concentration of fibers is small, which is the case for most fiber materials used in acoustics.

From this one computes by numerical integration for $d<0.1$.

$$
\left\langle\sigma^{2} \ln (\sigma)\right\rangle=0.446 .
$$

From Eqs. (27)-(34) one finds the mean flux

$$
\langle\Psi\rangle=\frac{1}{4 \pi \eta} \frac{\partial p}{\partial z} b^{4}\left[-1.280 \ln \left(\frac{1}{d}\right)-2 d+1.474\right],
$$

where a term with $d$ squared has been neglected. The mean velocity is

$$
\langle u\rangle=\frac{\Psi}{b^{2}}=-\frac{b^{2}}{4 \pi \eta} \frac{\partial p}{\partial z}\left[1.280 \ln \left(\frac{1}{d}\right)+2 d-1.474\right] .
$$

From this and Eq. (1) one gets the resistivity for flow along randomly placed fibers $R_{\mathrm{lr}}$,

$$
R_{\mathrm{lr}}=\frac{4 \pi \eta}{b^{2}[1.280 \ln (1 / d)+2 d-1.474]} .
$$

Sangani and $\mathrm{Yao}^{5}$ have computed numerically the permeability for 16 cylinders placed randomly. For $d=0.1$, they give the permeability $4.9 \pm 0.7$, where 0.7 is the standard deviation. From Eq. (26) and (37) one finds the permeability 4.2. From this one can conclude that the above procedure is sufficiently accurate for the present purpose.

\section{FLOW PERPENDICULAR TO FIBERS}

Flow perpendicular to parallel cylinders is now considered. We treat parallel cylinders in two cases: placed in a square lattice and randomly placed. The last case is more important than the first one, but the case of the square lattice is much easier than the random one, and the methods used for the first case can be extended to the random case.

For acoustic material, we are only interested in volume concentrations of fibers smaller than 0.02 in most cases, which makes some of the computations easier.

The resistivity of the two models is found by solving the Navier-Stokes equation for incompressible flow. We use Stokes' stream-function $\psi$, and find the velocity from

$$
u_{x}=\frac{\partial \psi}{\partial y}, \quad u_{y}=-\frac{\partial \psi}{\partial x} .
$$

This procedure secures that the condition of incompressibility Eq. (3) is fulfilled. The stream functions can be computed from

$$
\nabla^{2} \psi=\omega,
$$

where the vorticity $\omega$ is defined by

$$
\omega=\frac{\partial u_{x}}{\partial y}-\frac{\partial u_{y}}{\partial x},
$$

and

$$
\nabla^{2} \omega=0 .
$$

\section{A. Square lattice}

This case has been treated by Sangani and Acrivos. ${ }^{16}$ We assume the flow is in the direction of the $x$ axis in Fig. 2. The boundary conditions that follow from the symmetry of the flow are

$$
\begin{aligned}
& \omega=0, \quad \psi=1 \quad \text { on } \quad A B, \\
& \frac{\partial \psi}{\partial x}=\frac{\partial \omega}{\partial x}=0 \quad \text { on } \quad B C .
\end{aligned}
$$

$\psi$ is constant on $A B$ because $u_{y}=0$, which follows from the symmetry of the flow. We set $\psi=1$ in order to simplify the equations. This is not dimensionally correct, but the resistivity in not influenced by this. We could have set $\psi=$ constant and secured the dimensions. Nonslip is assumed on the cylinders, therefore

$$
\psi=\frac{\partial \psi}{\partial r}=0 \quad \text { on the cylinder surface. }
$$

The boundary conditions equations (42)-(44) are explained in the Appendix. Sangani and Acrivos found the resistivity

$R_{\mathrm{cs}}=\frac{4 \pi \eta}{b^{2}\left[\ln \left(d^{-1 / 2}\right)-0.738+d-0.887 d^{2}+2.03 d^{3}+O\left(d^{4}\right)\right]}$.

The following calculation by Kuwabara ${ }^{17}$ shows a simpler way to reach the same result valid for small concentration of cylinders. Kuwabara ${ }^{17}$ replaced the square perimeter of the unit cell with a circle. The area of the circle equals the area of the unit cell, and one requires zero vorticity on the perimeter of the circle. One now has a flow problem for two concentric cylinders with $\omega=0$ on the outer cylinder and Eq. (44) on the inner cylinder. Only one boundary condition is given on the outer cylinder, therefore the solution contains an integration constant of no importance for the calculation 
of resistivity. The boundary condition $\omega=0$ cannot be derived directly from Eqs. (42) and (43), but is based on the physical reasonable assumption that the vorticity is zero close to the perimeter of the unit cell. Kuwabara ${ }^{17}$ used the same procedure; it is repeated here in order to make the following procedure for random fibers more easy to understand. From this he found the resistivity

$$
R_{\mathrm{cs}}=\frac{4 \pi \eta}{b^{2}\left[\ln \left(d^{-1 / 2}\right)-\frac{3}{4}+d-\frac{1}{4} d^{2}\right]} .
$$

In acoustic fiber materials where the concentration is mostly less than 0.02 , there is little difference between the two equations (45) and (46).

In the following we use Kuwabara's approach modified in a way that can be extended to random distributions of fibers. We compute the resistivity by calculating the energy dissipation.

$\mathrm{Lamb}^{18}$ has treated a problem of flow around a cylinder. From this one sees that the vorticity is

$$
\omega=A\left[\frac{1}{r}-\frac{r}{\rho^{2}}\right] \sin \nu,
$$

where $r$ is the radius in a polar coordinate system and $\nu$ is the angle. The direction of flow is determined by $\nu=0 . \rho$ is the radius of the cylinder where the vorticity is zero. $A$ is an integration constant. Equations (39) and (47) can be integrated to give

$$
\begin{aligned}
\psi= & A\left[\frac{r}{2} \ln \frac{r}{a}-\frac{r^{3}}{8 \rho^{2}}+\left(\frac{a^{2}}{4 \rho^{2}}-\frac{1}{4}\right) r\right. \\
& \left.+\left(\frac{a^{2}}{4}-\frac{a^{4}}{8 \rho^{2}}\right) \frac{1}{r}\right] \sin \nu,
\end{aligned}
$$

when one requires that the velocity is zero on the cylinder. The pressure can be found from Lamb. ${ }^{18}$ One gets

$$
p=A \eta\left(\frac{1}{r}+\frac{r}{\rho^{2}}\right) \cos \nu .
$$

The macroscopic gradient equals the pressure drop over the cell divided by the cell diameter, thus the macroscopic gradient is

$$
\langle\nabla p\rangle=A\left(2 \eta / \rho^{2}\right) .
$$

This equation is used to find the integration constant

$$
A=\rho^{2}\langle\nabla p\rangle / 2 \eta .
$$

In order to find the resistivity, we compute the power that flows into the cell through the cylinder surface surrounding a fiber. The power is

$$
P=-\oint p \mathbf{u} \cdot \mathbf{n} d S,
$$

where $\mathbf{n}$ is the normal to the surface. From this equation and Eqs. (38), (48), (49), and (51), one gets the power per length

$$
\frac{P}{l}=\frac{\pi \rho^{4}\langle\nabla p\rangle^{2}}{4 \eta}\left[\ln \frac{\rho}{a}-\frac{3}{4}+\frac{a^{2}}{\rho^{2}}-\frac{a^{4}}{4 \rho^{4}}\right],
$$

where $l$ is the length of the cylinder integrated over. The energy dissipated per volume equals

$$
\frac{P}{l b^{2}}=\frac{\langle\nabla p\rangle^{2}}{R} .
$$

From Eqs. (53) and (54) one finds the resistivity of Eq. (46). This shows that the energy method gives the same result as Kuwabara's ${ }^{17}$ method.

\section{B. Random lattice}

The resistivity against flow perpendicular to parallel fibers placed randomly will be computed from the power dissipated in the air. We use the Voronoi polygons to compute the resistivity in this case. From Eq. (54)

$$
R=\frac{\langle\nabla p\rangle^{2} l b^{2}}{\langle P\rangle},
$$

where $\langle P\rangle$ is the mean power per cell.

The power in Eq. (53) can be written in terms of the area $S$ of the circle. The power becomes

$P=\frac{\langle\nabla p\rangle^{2} l}{4 \pi \eta}\left[\frac{1}{2} S^{2} \ln \left(\frac{S}{\pi a^{2}}\right)-\frac{3}{4} S^{2}+\pi a^{2} S-\frac{1}{4}\left(\pi a^{2}\right)^{2}\right]$.

We replace the Voronoi polygons with circles with the same area as the polygons. It is convenient to use the variable $\sigma$, i.e., the area of the circles divided by the mean area per fiber. The power can be written

$$
\begin{aligned}
P= & \frac{\langle\nabla p\rangle^{2} l b^{4}}{4 \pi \eta}\left[\frac{1}{2} \sigma^{2} \ln \sigma+\frac{1}{2} \sigma^{2} \ln \left(\frac{1}{d}\right)-\frac{3}{4} \sigma^{2}+d \sigma\right. \\
& \left.-\frac{1}{4} d^{2}\right] .
\end{aligned}
$$

The mean value of the power over the ensemble of Voronoi polygons can be found from the average values of $\sigma$ and function of $\sigma$ as given in Eqs. (31)-(34). From this one finds the average value of the power:

$$
\langle P\rangle=\frac{\langle\nabla p\rangle^{2} l b^{4}}{4 \pi \eta}\left[0.640 \ln \left(\frac{1}{d}\right)-0.737+d\right],
$$

where a term with the concentration squared has been neglected. From this we find the resistivity

$$
R_{\mathrm{cr}}=\frac{4 \pi \eta}{b^{2}[0.640 \ln (1 / d)-0.737+d]} .
$$

From this one can compute the permeability defined in Eq. (25). For $d=0.1$ one finds from (59) the permeability 2.09 . Sangani and $\mathrm{Yao}^{5}$ have numerically computed the permeability for 16 cylinders placed randomly; for $d=0.1$ they give the permeability $1.67 \pm 0.12$. The last number is the standard deviation. The difference between these two permeabilities is more than could be expected from the standard deviation 0.12 . They also give 1.79, which was based on Howells, analytic calculations. Neither Howell ${ }^{4}$ nor Sangani and $\mathrm{Yao}^{5}$ give a closed formula with which Eq. (59) could be compared. 


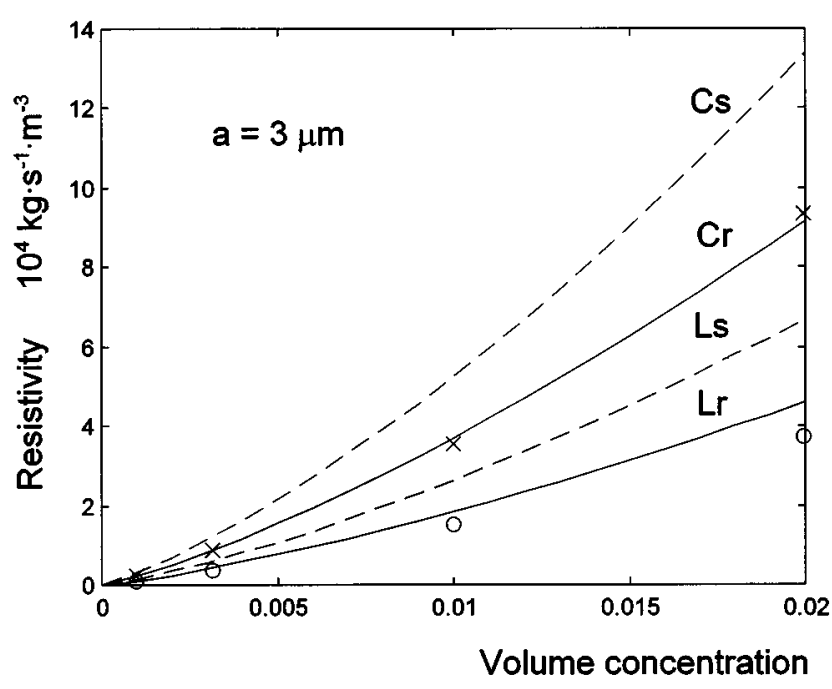

FIG. 4. The resistivity for parallel cylinders as a function of volume concentration of cylinders. The radius of the cylinders (fibers) is assumed to be $3 \mu \mathrm{m}$, which is a value typical for glass wool. For fixed value of concentration the resistivity is inversely proportional with the square of the radius. Cs means cross flow in a square lattice. $\mathrm{Cr}$ means cross flow in a random lattice. Lc means longitudinal flow in a square lattice. Lr means longitudinal flow in a random lattice. The points were calculated from Howells ${ }^{4}$ who used a different method to calculate the resistivity.

\section{DISCUSSION}

The resistivity of a set of parallel plates with equal distances is six times the resistivity of randomly placed plates for the same mean density, as can be seen from (8) and (11). In the case of parallel cylinders, the resistivity is also greater when the cylinders are placed regularly in a lattice, but the difference is not as great as in the one-dimensional case. This can be seen from Fig. 4, which shows the resistivity as a function of volume concentration of cylinders. The resistivity is shown for a cylinder radius of $3 \mu \mathrm{m}$. The resistivity in the case of other cylinder (fiber) diameters can be found from the figure, because the resistivity is inversely proportional to the square of the cylinder radius when the concentration is constant. The line marked Cs is the resistivity for cross flow through a square lattice. $\mathrm{Cr}$ means cross flow through random placed cylinders. Ls is longitudinal flow in the case of square lattice, and $\mathrm{Lr}$ is longitudinal flow for random placement. The points are calculated from Howells ${ }^{4}$ who used another method to calculate the resistivity.

The volume density of the fibers equals the mass density of glass wool divided by the mass density of the glass itself. A typical value of concentration for light glass wool with a density of $16 \mathrm{~kg} / \mathrm{m}^{3}$ is

$$
d=\frac{16 \mathrm{~kg} / \mathrm{m}^{3}}{2550 \mathrm{~kg} / \mathrm{m}^{3}}=0.0063 \text {. }
$$

\section{CONCLUSION}

Explicit formulae for resistivity to airflow through parallel cylinders placed randomly have been developed in a new way, both for flow parallel with Eq. (37) and perpendicular to Eq. (59) the cylinders. The formulae are valid for

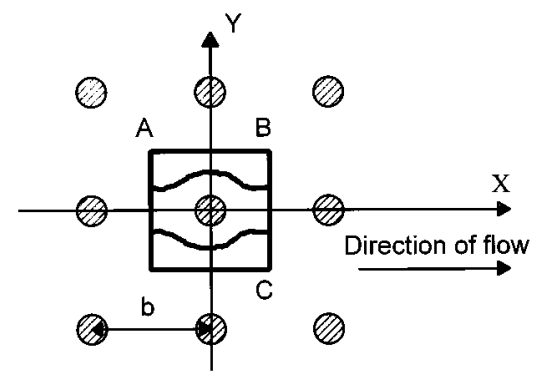

FIG. A1. The streamlines for flow in a square lattice. The flow is assumed to be in the direction of the $x$ axis. The velocity vector is symmetric to reflexion in the $x$ axis, and symmetric to reflexion in the $y$ axis and time inversion. The flow is periodic with translations $b$ in the direction of the $x$ axis and $y$ axis.

the densities of cylinders (fibers) one finds in real fiber material such as glass wool. The results deviate less than $20 \%$ from earlier calculations by other authors.

\section{APPENDIX: EXPLANATIONS OF BOUNDARY CONDITIONS FOR SQUARE LATTICE}

Figure A1 shows streamlines in a unit cell. The flow is in the direction of the $x$ axis. The velocity is periodic with translations in the directions of the $x$ and $y$ axis, the period being equal to $b$, the distance between the axes of two neighbor cylinders. The velocity vector is symmetric to reflection in the $x$ axis and the line $A B$. Therefore, the velocity vector on $A B$ must be directed along $A B$. That means $u_{y}(x, b / 2)=0$, and $\partial u_{y}(x, b / 2) / \partial x=0$. The velocity must be maximum on $A B$, which means $\partial u_{x} / \partial y=0$. From the definition of vorticity Eq. (40) then follows the first part of Eq. (42). The velocity component perpendicular to $A B$ is zero. According to Eq. (38) this is fulfilled if $\psi$ is a constant on $A B$; this is the second part of Eq. (42).

The velocity vector is symmetric to reflection in the $y$ axis and time reflection. The line $B C$ is also a symmetry line for reflection and time inversion. The boundary conditions on line $B C$ can be deduced from this symmetry. Due to symmetry, the velocity is perpendicular to $B C$, which means that the velocity component in the $y$ direction is zero; from Eq. (38) follows then the first of Eq. (43).

In order to prove the second boundary condition on $B C$, we first calculate some derivatives. The incompressibility condition is

$$
\frac{\partial u_{x}}{\partial x}+\frac{\partial u_{y}}{\partial y}=0 .
$$

On $B C, u_{y}=0$. Therefore, on $B C, \partial u_{y} / \partial y=0$, and from Eq. (A1), $\partial u_{x} / \partial x=0$. From this follows that on $B C$, $\partial^{2} u_{x} / \partial y \partial x=0$. However, $u_{y}(\xi+b / 2, y)$ is an uneven function of $\xi$ due to the symmetry. Therefore, $\partial^{2} u_{y} / \partial x^{2}=0$. From the definition of the vorticity and the value of the partial derivatives, one gets on $B C$

$$
\frac{\partial \omega}{\partial x}=\frac{\partial^{2} u_{x}}{\partial x \partial y}-\frac{\partial^{2} u_{y}}{\partial^{2} x}=0 .
$$

This is the second part of Eq. (43). 
${ }^{1}$ M. E. Delany and E. N. Bazley, "Acoustical properties of fibrous absorbent materials," Appl. Acoust. 3, 105-116 (1970).

${ }^{2} \mathrm{~V}$. Tarnow, "Measurement of sound propagation in glass wool," J. Acoust. Soc. Am. 97(4), 2272-2281 (1995).

${ }^{3}$ B. T. Aström, R. B. Pipes, and S. G. Advani, "On flow through aligned fiber beds and its application to composites processing," J. Compos. Mater. 26(9), 1351-1373 (1992).

${ }^{4}$ I. D. Howells, "Drag due to motion of a Newtonian fluid through a sparse random array of small fixed rigid objects," J. Fluid. Mech. 64, 449-475 (1974).

${ }^{5}$ A. S. Sangani and C. Yao, "Transport processes in random arrays of cylinders. II. Viscous flow,' Phys. Fluids 31(9), 2435-2444 (1988).

${ }^{6} \mathrm{~V}$. Tarnow, "Compressibility of air in fibrous materials," J. Acoust. Soc. Am. 99, 3010-3017 (1996).

${ }^{7}$ L. D. Landau and E. M. Lifshitz, Fluid Mechanics (Pergamon, New York, 1966), Secs. 15 and 17.

${ }^{8}$ A. D. Pierce, Acoustics (Acoustical Society of America, Woodbury, NY, 1989), p. 513.

${ }^{9}$ J. M. Ziman, Models of Disorder (Cambridge U.P., Cambridge, UK, 1979), p. 39.

${ }^{10}$ E. M. Sparrow and A. L. Loeffler, Jr., "Longitudinal laminar flow be- tween cylinders arranged in regular array," AIChE J. 5(3), 325-330 (1959).

${ }^{11}$ A. J. Dekker, Solid State Physics (Prentice-Hall, Englewood Cliffs, NJ, 1962), p. 269.

${ }^{12}$ A. L. Berdichevsky and Z. Cai, "Preform permeability predictions by self-consistent method and finite element simulation," Polym. Compos. 14(2), 132-143 (1993)

${ }^{13} \mathrm{~F}$. Aurenhammer, "Vorohoi diagrams-A survey of a fundamental geometric data structure," ACM Comput. Surveys 23(3), 345-405 (1991).

${ }^{14}$ D. Weaire, J. P. Kermode, and J. Wejchert, "On the distribution of cell areas in a Voronoi network,' Philos. Mag. B 53(5), L101-L105 (1986).

${ }^{15} \mathrm{P}$. N. Andrade and M. A. Fortes, "Distribution of cell volumes in a Voronoi partition," Philos. Mag. B 58(6), 671-674 (1988).

${ }^{16}$ A. S. Sangani and A. Acrivos, "Slow flow past periodic arrays of cylinders with application to heat transfer," Int. J. Multiphase Flow 8, 193206 (1982).

${ }^{17} \mathrm{~S}$. Kuwabara, "The forces experienced by randomly distributed parallel circular cylinders or spheres in a viscous flow at small Reynolds numbers," J. Phys. Soc. Jpn. 14, 527-532 (1959).

${ }^{18}$ H. Lamb, Hydrodynamics (Cambridge U.P., New York, 1993), Sec. 341. 